\title{
Wavelength selective quantum dot infrared photodetector with periodic metal hole arrays
}

\author{
Chi-Yang Chang, ${ }^{\text {a) }}$ Hsu-Yu Chang, Chia-Yi Chen, Ming-Wei Tsai, \\ Yi-Tsung Chang, and Si-Chen Lee \\ Department of Electrical Engineering, Graduate Institute of Electronics Engineering, National Taiwan \\ University, Taipei, Taiwan 106, Republic of China
}

Shiang-Feng Tang

National Science Council of the Republic of China, Taoyuan, Taiwan 325, Republic of China

(Received 14 August 2007; accepted 28 September 2007; published online 17 October 2007)

\begin{abstract}
In this letter, the effect of extraordinary transmission of periodic metal hole arrays is directly integrated into the quantum dot infrared photodetector with broadband response. It is found that the detector response is strongly modulated by the extraordinary transmission from the excitation of surface plasmon. ㅇ 2007 American Institute of Physics. [DOI: 10.1063/1.2800378]
\end{abstract}

Surface plasmon was widely explored in the field of surface science following the pioneering work of Ritchie in the 1950s. ${ }^{1}$ In 1998, Ebbesen et al. reported that metal films with two-dimensional subwavelength periodic perforated hole arrays exhibit extraordinary optical transmission because of surface plasmon. ${ }^{2,3}$ Afterwards, the surface plasmon properties have attracted researchers due to their potential applications in subwavelength photolithography, near-field microscopy, wavelength-tunable filter, and optical modulators. ${ }^{4,5}$ In addition, self-assembled InAs quantum dots on the GaAs substrate prepared by molecular beam epitaxy have drawn much attention in recent years. It is in great need for a variety of applications ranging from night vision and battle recognition systems to chemical spectroscopy and remote sensing. ${ }^{6,7}$ Compared to the quantum well infrared photodetector and superlattice infrared photodetector, the improved properties of quantum dot infrared photodetector (QDIP) have low dark current, polarization independent, broadband detection spectrum, and high temperature operation.

There are two transmission windows for electromagnetic wave propagation in atmosphere, i.e., 3-5 and 8-12 $\mu \mathrm{m}$. Typically, two kinds of photodetectors are needed to detect these two band signals. In this work, we choose a single QDIP with broadband response ranging from 2.5 to $12 \mu \mathrm{m}$ incorporated with two periodic metal hole arrays to achieve this purpose. By choosing periodic hole arrays with appropriate lattice constant and directly integrating into the back side of the QDIP, the response peak of the QDIP will be selected by the extraordinary transmission of the surface plasmons from the periodic metal hole array.

The device structure of a ten layer $\mathrm{In}_{0.1} \mathrm{Ga}_{0.9} \mathrm{As} / \mathrm{InAs} / \mathrm{In}_{0.1} \mathrm{Ga}_{0.9} \mathrm{As} / \mathrm{GaAs}$ QDIP is shown in Fig. 1(a). The fabrication processes of QDIP were described elsewhere. ${ }^{8}$ Afterwards, the pattern was defined to the back side of the QDIP and 100-nm-thick Ag metal film was thermally deposited and lifted off to form the periodic hole arrays, as shown in Fig. 1(b). Table I lists the lattice constants and hole dimension of the metal array of four samples, A-D. The QDIPs in these four samples were from the same run, as shown in Fig. 1(a). Sample A is "blank" indicating no metal film on the back of QDIP; its photoresponse is used as a

\footnotetext{
a)Electronic mail: r94943061@ntu.edu.tw
}

reference to compare with those of other three samples. The array parameters are chosen to match the wavelength window of the QDIP. The response of QDIPs was measured at $10 \mathrm{~K}$ using Bruker IFS $66 \mathrm{v} / \mathrm{s}$ system. The wave number resolution of the measurement was $8 \mathrm{~cm}^{-1}$. The IR light source is normally incident on the back side of the QDIP with perforated $\mathrm{Ag}$ hole arrays.

As surface plasmon wave propagated at interface of a two-dimensional lattice, the momentum conservation law of surface plasmon is given by ${ }^{3}$

$$
k_{\mathrm{sp}}=k_{x}+i G_{x}+j G_{y},
$$

where $k_{\mathrm{sp}}=(\omega / c) \sqrt{\varepsilon_{d} \varepsilon_{m} /\left(\varepsilon_{d}+\varepsilon_{m}\right)}$ is the surface plasmon wavevector, $\mathbf{k}_{x}=\left|\mathbf{k}_{0}\right| \sin \theta,\left|\mathbf{k}_{0}\right|=2 \pi / \lambda$ is the wavevector of the incident radiation, $\lambda$ is the wavelength in vacuum, and $\theta$ is the angle between the incident radiation and the $z$ direction. $\varepsilon_{d}$ and $\varepsilon_{m}$ are the dielectric constants of the dielectric material and metal, respectively. The $G_{x}=(4 \pi / \sqrt{3} a)[(1 / 2) \hat{x}$ $+(\sqrt{3} / 2) \hat{y}]$ and $G_{y}=(4 \pi / \sqrt{3} a)[(1 / 2) \hat{x}-(\sqrt{3} / 2) \hat{y}]$ are the reciprocal lattice vectors associated with the hexagonal array, where $a$ is the lattice constant. For normally incident light, $k_{x}=0$, Eq. (1) is reduced to

$$
\lambda_{\mathrm{sp}}=a\left[\frac{4}{3}\left(i^{2}+i j+j^{2}\right)\right]^{-1 / 2}\left(\frac{\varepsilon_{d} \varepsilon_{m}}{\varepsilon_{d}+\varepsilon_{m}}\right)^{1 / 2} .
$$

Figures 2(a) and 2(b) show the normally incident transmission spectra of GaAs wafer with hexagonally ordered $\mathrm{Ag}$ hole arrays with lattice constant $a=1.7 \mu \mathrm{m}$ and diameter $d$ $=1.0 \mu \mathrm{m}$ (the same as metal array on sample B) and lattice constant $a=3.4 \mu \mathrm{m}$ and diameter $d=1.4 \mu \mathrm{m}$ (the same as metal array on sample C), respectively. The dielectric constant of GaAs is 10.89. It is clear that the transmission peaks of the spectra are roughly at 5 and $9.9 \mu \mathrm{m}$, respectively, which are close to the theoretical values predicted by Eq. (2), i.e., 4.9 and $9.7 \mu \mathrm{m}$, respectively. These peaks are due to the $( \pm 1,0),(0, \pm 1),(1,-1)$, and $(-1,1) \mathrm{Ag} / \mathrm{GaAs}$ degenerate surface plasmon modes. Figure 3 illustrates the responsivities of the QDIP samples A-C measured at $10 \mathrm{~K}$ and biased at $+1.8 \mathrm{~V}$. The response window of sample $\mathrm{A}$ without any metal array on the back is very wide ranging from 2.5 to $12 \mu \mathrm{m}$. However, the peak responses of samples B and $\mathrm{C}$ are very narrow which are modulated by the extraor- 

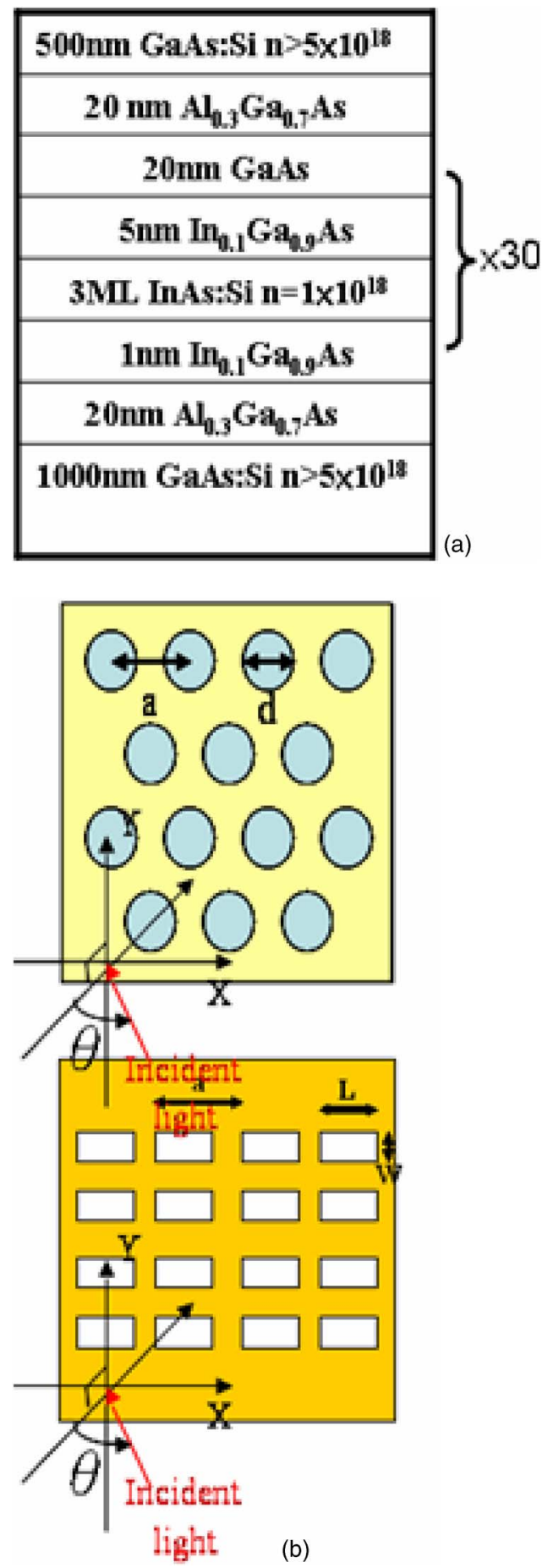

FIG. 1. (Color online) (a) The device structure of the QDIP. (b) Definition of the parameters of hexagonal hole and rectangular hole arrays.

dinary transmission of metal arrays, as shown in Figs. 2(a) and 2(b). However, in the practical application, the operating window of QDIP is in a specific range such as far infrared $(8-12 \mu \mathrm{m})$. Thus, the Ag film with patterned rectangular hole arrays is chosen to achieve the target.

Figure 4 shows the comparison of normally incident transmission spectra of the GaAs wafer with hexagonal holes with $a=3.4 \mu \mathrm{m}$ and $d=1.4 \mu \mathrm{m}$, square holes with $a$ $=3.0 \mu \mathrm{m}$ and $L=1.3 \mu \mathrm{m}$, and rectangular holes with $a$ $=3.0 \mu \mathrm{m}$ and $L \times W=2 \times 1 \mu \mathrm{m}^{2}$, respectively. It is found that the transmission peaks of the hexagonal hole and square hole arrays are roughly the same at $10 \mu \mathrm{m}$, but the full width at half maximum (FWHM) of square holes is broader than Downloaded 04 Mar 2009 to 140.112 .113 .225 . Redistribution subje
TABLE I. The device parameters of samples A-D.

\begin{tabular}{cl}
\hline \hline Sample & Specification \\
\hline A & Blank \\
& Hexagonal holes arrays, \\
& lattice constant $a=1.7 \mu \mathrm{m}$, hole \\
& diameter $d=1.0 \mu \mathrm{m}$ \\
B & Hexagonal holes arrays, \\
& lattice constant $a=3.4 \mu \mathrm{m}$, hole \\
& diameter $d=1.4 \mu \mathrm{m}$ \\
C & Rectangular holes arrays, \\
& lattice constant \\
& $a=3.0 \mu \mathrm{m}, L \times W=2 \times 1 \mu \mathrm{m}^{2}$ \\
\hline
\end{tabular}

that of hexagonal ones. The percentage of the total area of square holes is $19 \%$ which is larger than that of hexagonal holes $(15 \%)$. Therefore, the effect of radiation damping caused by the scattering of surface plasmon with the apertures on metal film would be enlarged. In case of rectangular holes, the transmission peak redshifts from 10 to $10.6 \mu \mathrm{m}$. It is due to the strong coupling of the localized surface plasmon on the long edges of rectangular holes. ${ }^{9}$ On the other hand, the FWHM of rectangular holes is broader than that of square holes. For rectangular holes, the surface plasmon tends to couple strongly with the local charge dipole oscillations between the long edges. ${ }^{10}$ In other words, $Y$-polarized
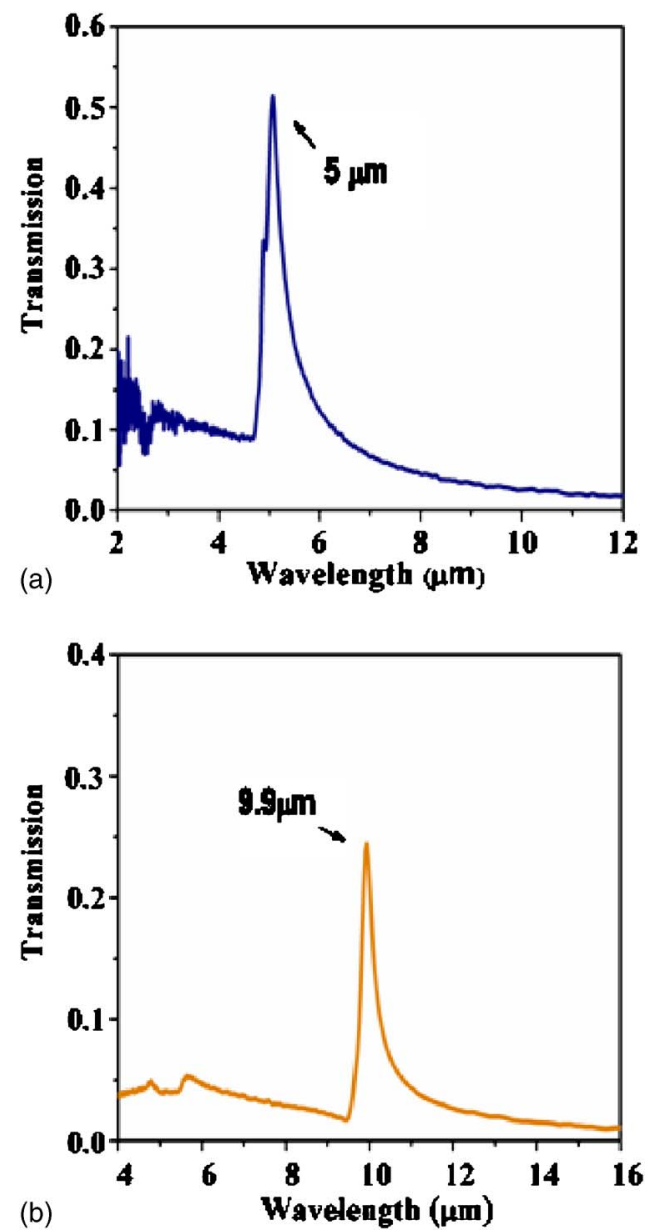

FIG. 2. (Color online) Normally incident transmission spectra of the GaAs wafer with hexagonally patterned $\mathrm{Ag}$ film of (a) lattice constant $a$ $=1.7 \mu \mathrm{m}$ and diameter $d=1.0 \mu \mathrm{m}$ and (b) lattice constant $a=3.4 \mu \mathrm{m}$ and diameter $d=1.4 \mu \mathrm{m}$.

to AIP license or copyright; see http://apl.aip.org/apl/copyright.jsp 


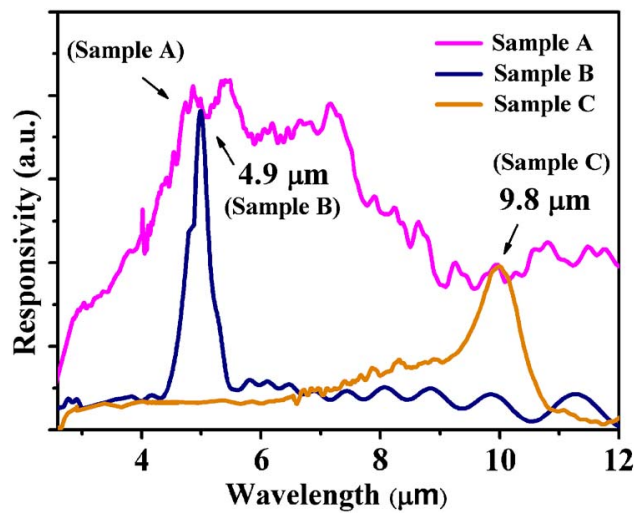

FIG. 3. (Color online) Infrared responsivities of the samples A-C.

light perpendicular to the long edge of the rectangular holes tends to induce charge dipole oscillation between the long edges ( $y$ axis). Therefore, the effective scattering length for rectangular holes is $2 \mu \mathrm{m}$ larger than that for square holes $(1.3 \mu \mathrm{m})$. Thus, the rectangular holes with $a=3.0 \mu \mathrm{m}$ and $L \times W=2 \times 1 \mu \mathrm{m}^{2}$ are chosen in this experiment. Figure 5 shows the comparison of the responsivities between samples $\mathrm{A}$ and $\mathrm{D}$ at the same bias of $+1.8 \mathrm{~V}$ and a temperature of $10 \mathrm{~K}$. It is clear that the responsivity with $\mathrm{Ag}$ rectangular hole arrays is much broader than that of the hexagonally ordered hole arrays. The extra peak is caused by the $(1,1)$

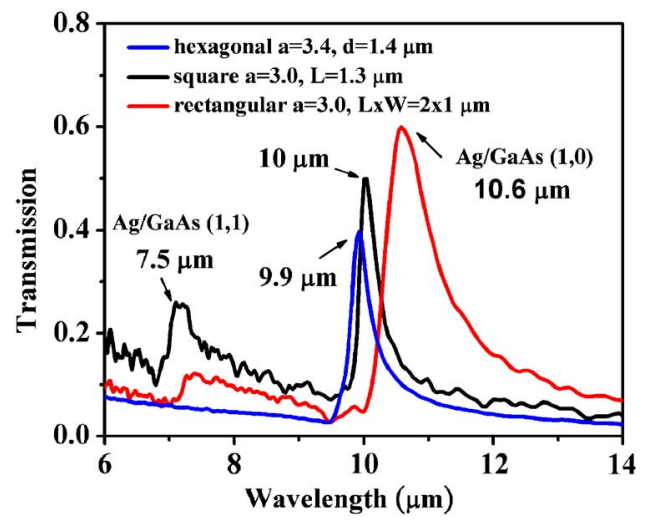

FIG. 4. (Color online) The comparison of normally incident transmission spectra of the GaAs wafer with hexagonal holes with $a=3.4 \mu \mathrm{m}$ and $d$ $=2.0 \mu \mathrm{m}$, square holes with $a=3.0 \mu \mathrm{m}$ and $L=1.3 \mu \mathrm{m}$, and rectangular holes with $a=3.0 \mu \mathrm{m}$ and $L \times W=2 \times 1 \mu \mathrm{m}^{2}$.

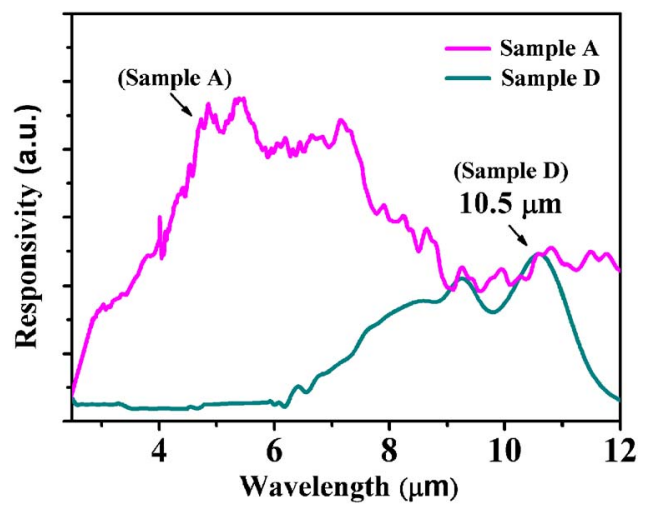

FIG. 5. (Color online) The comparison of normally incident transmission spectra of the samples A and D.

$\mathrm{Ag} / \mathrm{GaAs}$ mode at $7.3 \mu \mathrm{m}$, as shown in Fig. 4. Thus, not only the specifications but also the shape of the holes can be selected to achieve the anticipated results.

In summary, the characteristic of surface plasmon is successfully applied to the wavelength selective QDIP. By choosing the lattice constant and the shape of the hole of the metal array, the response of the QDIP can be modulated by the extraordinary transmission from the excitation of surface plasmon. By utilizing this coupling scheme, it opens the way to fabricate multicolor infrared photodetectors and to manufacture infrared photodetectors in focal plane array.

This work is mainly supported by Chung-shan Institute of Science and Technology. The authors would also like to thank the National Science Council of the Republic of China, Taiwan, for partial support under Contract No. NSC 952215-E-002-042.

${ }^{1}$ R. H. Ritchie, Phys. Rev. 106, 874 (1957).

${ }^{2}$ T. W. Ebbesen, H. J. Lezec, H. F. Ghaemi, T. Thio, and P. A. Wolff, Nature (London) 391, 667 (1998).

${ }^{3}$ H. F. Ghaemi, T. Thio, D. E. Grupp, T. W. Ebbesen, and H. J. Lezec, Phys. Rev. B 58, 6779 (1998).

${ }^{4}$ J. R. Sambles, Nature (London) 391, 641 (1998)

${ }^{5}$ T. Thio, H. J. Lezec, and T. W. Ebbesen, Physica B 279, 90 (2000).

${ }^{6}$ S. Raghavan, P. Rotella, A. Stintz, B. Fuchs, S. Krishna, C. Morath, D. A. Cardimona, and S. W. Kennerly, Appl. Phys. Lett. 81, 1369 (2002).

${ }^{7}$ H. Kaplan, Photonics Spectra 33, 104 (1999).

${ }^{8}$ S. F. Tang, S. Y. Lin, and S. C. Lee, Appl. Phys. Lett. 78, 2428 (2001).

${ }^{9}$ R. Gordon and A. G. Brolo, Opt. Express 13, 1933 (2005).

${ }^{10}$ M.-W. Tsai, T.-H. Chuang, H.-Y. Chang, and S.-C. Lee, Appl. Phys. Lett. 89, 093102 (2006). 